\title{
Green Tea Polyphenols Attenuated Glutamate Excitotoxicity via Antioxidative and Antiapoptotic Pathway in the Primary Cultured Cortical Neurons
}

\author{
Lin Cong, ${ }^{1,2}$ Chang Cao, ${ }^{1,3}$ Yong Cheng, ${ }^{4}$ and Xiao-Yan Qin ${ }^{1,3}$ \\ ${ }^{1}$ Beijing Engineering Research Center of Food Environment and Health, Minzu University of China, Beijing 100081, China \\ ${ }^{2}$ Department of General Surgery, Peking Union Medical College Hospital, Chinese Academy of Medical Sciences and \\ Peking Union Medical College, Beijing 100730, China \\ ${ }^{3}$ College of Life \& Environmental Science, Minzu University of China, Beijing 100081, China \\ ${ }^{4}$ Laboratory of Neurobiology and State Key Laboratory of Biomembrane and Membrane Biotechnology, College of Life Sciences, \\ Peking University, Beijing 100871, China \\ Correspondence should be addressed to Yong Cheng; chengy4@mail.nih.gov and Xiao-Yan Qin; zhongsijia01@163.com
}

Received 31 July 2015; Accepted 13 September 2015

Academic Editor: Silvana Hrelia

Copyright (C) 2016 Lin Cong et al. This is an open access article distributed under the Creative Commons Attribution License, which permits unrestricted use, distribution, and reproduction in any medium, provided the original work is properly cited.

\begin{abstract}
Green tea polyphenols are a natural product which has antioxidative and antiapoptotic effects. It has been shown that glutamate excitotoxicity induced oxidative stress is linked to neurodegenerative diseases such as Alzheimer's disease and Parkinson's disease. In this study we explored the neuroprotective effect of green teen polyphenols against glutamate excitotoxicity in the primary cultured cortical neurons. We found that green tea polyphenols protected against glutamate induced neurotoxicity in the cortical neurons as measured by MTT and TUNEL assays. Green tea polyphenols were then showed to inhibit the glutamate induced ROS release and SOD activity reduction in the neurons. Furthermore, our results demonstrated that green tea polyphenols restored the dysfunction of mitochondrial pro- or antiapoptotic proteins Bax, Bcl-2, and caspase- 3 caused by glutamate. Interestingly, the neuroprotective effect of green tea polyphenols was abrogated when the neurons were incubated with siBcl-2. Taken together, these results demonstrated that green tea polyphenols protected against glutamate excitotoxicity through antioxidative and antiapoptotic pathways.
\end{abstract}

\section{Introduction}

Glutamate is a major neurotransmitter in the mammalian central nervous system. Glutamatergic neurons form the main excitatory system in the brain and play a pivotal role in many neurophysiological functions [1]. However, excessive release of glutamate can lead to neuronal dysfunction and cell death in a process now referred to as excitotoxicity [2]. Remarkably, glutamate excitotoxicity had been shown to play an important role in many neurodegenerative diseases including Alzheimer's disease (AD), Parkinson's disease (PD), and Huntington's disease (HD) [3]. Growing evidence suggests that the mitochondrial dysfunction mediated by glutamate excitotoxicity-induced oxidative stress was associated with both acute and chronic neurodegenerative disorders [4].
Moreover, it has been shown that glutamate excitotoxicity was involved in the pathogenesis of inflammatory pain [5]. In fact, reactive oxygen species have also been implicated in the cascade of events resulting from inflammatory pain $[5,6]$. Since glutamate excitotoxicity and oxidative stress are two common features for various neurodegenerative diseases [3] and inflammatory pain, searching for drugs or natural products targeting glutamate excitotoxicity and oxidative stress is a good strategy for potential treatments of neurodegenerative diseases and inflammatory pain.

Recently, a rapidly growing number of polyphenolic compounds with neuroprotective effects have been described [7]. The most discussed effects of polyphenols are the antioxidant properties. Dietary intake of polyphenols is known to attenuate oxidative stress and reduce the risk for related 
neurodegenerative diseases such as $\mathrm{AD}, \mathrm{PD}$, and $\mathrm{HD}$ [8]. Interestingly, green tea polyphenols have been found to be more antioxidant active than vitamin $\mathrm{C}$ and vitamin $\mathrm{E}$ [9]. Furthermore, green tea polyphenols were shown to protect against beta-amyloid toxicity in the primary cultured cortical neurons [10]. Further study confirmed the potential beneficial effect of green tea polyphenols in $\mathrm{AD}$ as green tea polyphenols demonstrated inhibition of acetylcholinesterase [11]. However, the effects of green tea polyphenols on excitotoxicity are still poorly understood.

In this study we have explored the potential protection of green tea polyphenols against glutamate excitotoxicity. We found that glutamate induced neurotoxicity in the primary cultured cortical neurons was inhibited by green tea polyphenols. We then showed that green tea polyphenols rescued glutamate induced oxidative stress. We further demonstrated that green tea polyphenols restored the dysfunction of proand/or antiapoptotic proteins Bax, caspase-3, and Bcl-2 caused by glutamate excitotoxicity. More interestingly, we used SiBcl-2 and showed that the neuroprotection of green tea polyphenols was abrogated with the treatment of SiBcl-2 in the neurons. Thus our results demonstrated that green tea polyphenols attenuated glutamate excitotoxicity via antioxidative stress and antiapoptotic pathway.

\section{Materials and Methods}

2.1. Primary Cortical Neuron Culture. Primary cortical neuron cultures were prepared as described previously [12]. Briefly, cortical tissues were dissected and dissociated. Then the cells were centrifuged and resuspended in DMEM with $10 \%$ fetal bovine serum (Invitrogen, CA, USA), 2 g/L HEPES (Invitrogen), $100 \mu \mathrm{g} / \mathrm{mL}$ penicillin, and $100 \mu \mathrm{g} / \mathrm{mL}$ streptomycin (Invitrogen). Cells were then plated in poly-L-lysinecoated (Sigma, St. Louis, Missouri) plates or coverslips. Each well was plated with $1 \times 10^{6}$ cells for 12 -well plates, $5 \times 10^{5}$ cells for 24 -well plates, and $1 \times 10^{5}$ cells for 96 -well plates. The cultured neurons were maintained in an incubator with $5 \% \mathrm{CO}_{2}$ and $95 \% \mathrm{O}_{2}$ at $37^{\circ} \mathrm{C}$ and were treated at 5-7 days in culture. $10 \mu \mathrm{M}$ cytosine arabinoside (Sigma) was added to the medium to inhibit the growth of glial cells after three days of culture.

2.2. Reagents and Treatments. The property of green tea polyphenols with $98 \%$ purity has been described previously [10]. Green tea polyphenols were added freshly into culture medium during treatments for $24 \mathrm{~h}$; then glutamate was added to the culture medium for $24 \mathrm{~h}$ to induce excitotoxicity in the primary cultured cortical neurons. In some experiments, the neurons were transiently transfected with $20 \mathrm{nM}$ siRNA oligonucleotides directed against $\mathrm{Bcl}-2$ ( $\mathrm{SiBcl}-2)$ or a scrambled control sequence (SiScr) (Invitrogen) for $24 \mathrm{~h}$ using Lipofectamine RNAiMAX (Invitrogen) according to the manufacturer's instruction.

2.3. Cell Viability and Death Assays. The cell viability was tested as described previously [13]. This was achieved by the neurons' ability to reduce the dye methyl thiazolyl tetrazolium (MTT, Sigma, MO) to its insoluble formazan, which has a purple color. Briefly, MTT was added to the 96well plates after various treatments in the primary cultured cortical neurons and the plate was maintained in an incubator for $2 \mathrm{~h}$. Then the supernatants were discarded and $150 \mu \mathrm{L}$ DMSO was added to each well to dissolve the formazan crystal. The absorption was recorded at $570 \mathrm{~nm}$ using a Bio-Rad plate reader (Thermo, MA, USA).

Cell death was measured by TUNEL assay as described previously [14]. Briefly, the primary cultured cortical neurons were fixed by $4 \%$ paraformaldehyde after treatments. Then the cells were permeabilized by $0.1 \%$ Triton X-100 with $0.1 \%$ sodium citrate on ice for 2 minutes. After that, in situ cell death detection kit was used for terminal deoxynucleotidyl transferase mediated dUTP nick-end labeling (TUNEL) staining to detect apoptotic cells as described by the manufacturer (Roche, IN, USA).

2.4. ROS and SOD Measurements. Reactive oxygen species (ROS) release in the primary cultured cortical neurons was measured by Cellular Reactive Oxygen Species Detection Assay Kit (Abcam). It used the cell permeant reagent $2^{\prime}, 7^{\prime}$ dichlorofluorescin diacetate (DCFDA); DCFDA is deacetylated by cellular esterases after diffused into the cells, which is later oxidized by ROS into $2^{\prime}, 7^{\prime}$-dichlorofluorescein (DCF). DCF is a highly fluorescent compound which can be detected by fluorescence spectroscopy. The experiments were performed according to the manufacturer's instruction.

Superoxide Dismutase (SOD) activity was assessed by measuring the dismutation of superoxide radicals generated by xanthine oxidase and hypoxanthine. The measurement of SOD activity in the neurons was achieved by Superoxide Dismutase Assay Kit (Cayman Chemical) as described by the manufacturer.

2.5. Western Blots. Western blots were used to test the protein levels after various treatments in the primary culture cortical neurons as described previously [15]. Briefly, the protein lysates were harvested from the neurons. Denatured protein samples with equal amounts were separated and transferred to PVDF membrane (Millipore, MA, USA). After blocking, the membranes were probed with primary antibodies followed by secondary antibodies. Protein bands were then detected by Bio-Rad ChemiDoc system (Bio-Rad, CA, USA) with the presence of enhanced chemiluminescence reagents. The following primary antibodies were used in the experiments: purified polyclonal rabbit anti- $\beta$-actin antibody (Santa Cruz, CA, USA), monoclonal rabbit anti-activated caspase-3 antibody (Cell Signaling, MA, USA), polyclonal rabbit anti-Bcl-2 antibody (Santa Cruz), and polyclonal rabbit anti-Bax antibody (Santa Cruz).

2.6. Statistical Evaluation. All data in the experiments are presented as means \pm SEM. Statistical significance $\left({ }^{*}\right.$ or ${ }^{\#} p<$ $0.05,{ }^{* *}$ or ${ }^{\# \#} p<0.01$, and ${ }^{* * *}$ or $\left.{ }^{\# \#} p<0.001\right)$ among groups was measured by one-way analysis of variance (ANOVA) followed by Tukey post hoc multiple comparisons tests. 


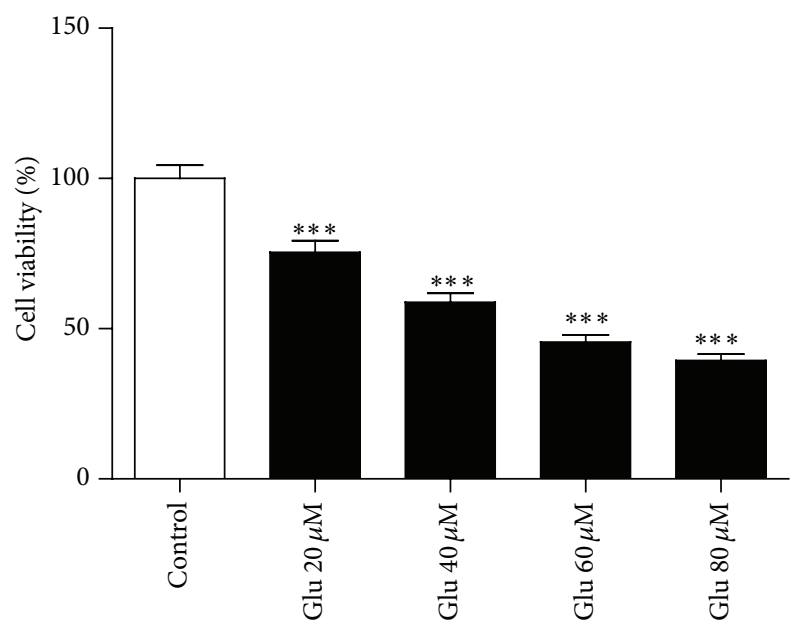

(a)

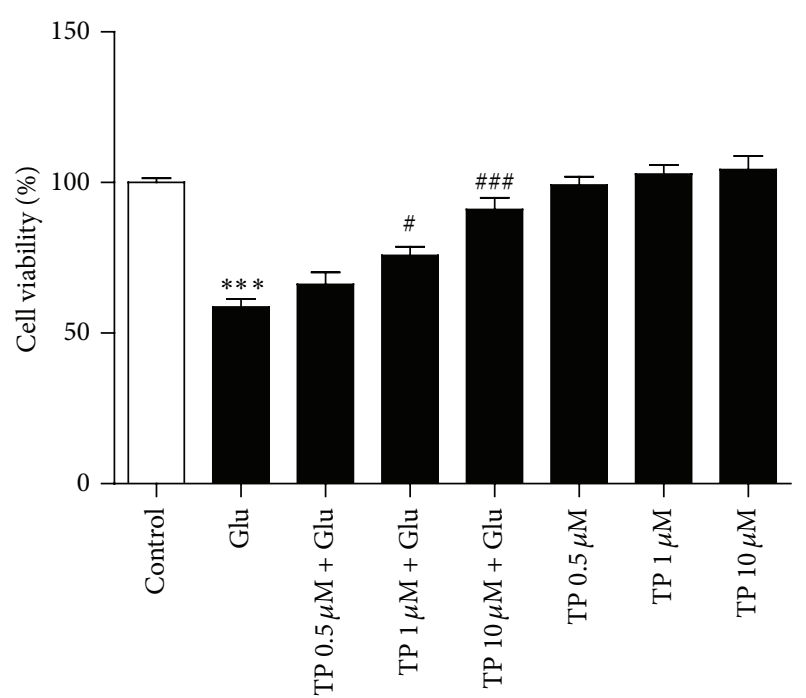

(b)

FIGURE 1: TP protected against glutamate (Glu) induced neurotoxicity in the primary cultured cortical neurons. (a) Glutamate reduced the cell viability at a dose dependent manner in the primary cultured cortical neurons as tested by MTT assay. (b) The glutamate induced reduction of cell viability was partially rescued by $1 \mu \mathrm{M}$ or $10 \mu \mathrm{M}$ TP in the neurons, while TP alone had no effect on the cell viability of the neurons. Glu: glutamate. At least three independent experiments were performed. Data shown represent one experiment. $n=5 /$ group. One-way analysis of variance (ANOVA) followed by Tukey post hoc multiple comparisons tests, $F_{(4,20)}=55.25$ for $(\mathrm{a}) ; F_{(7,32)}=29.69$ for $(\mathrm{b})$. ${ }^{* * *}$ compared to the control group and ${ }^{\#}$ or ${ }^{\# \#}$ compared to the Glu group.

\section{Results}

3.1. TP Attenuated Glutamate Excitotoxicity in Primary Cultured Cortical Neurons. In order to confirm the glutamate excitotoxicity, we treated the primary cultured cortical neurons with $20 \mu \mathrm{M}, 40 \mu \mathrm{M}, 60 \mu \mathrm{M}$, or $80 \mu \mathrm{M}$ glutamate for $24 \mathrm{~h}$; then MTT assay was used to measure the cell viability. As shown in Figure 1(a), glutamate reduced the cell viability at a dose dependent manner in the primary cultured cortical neurons. We next tested whether green tea polyphenols (TP) could rescue glutamate induced neurotoxicity in the neurons and pretreated the primary cultured cortical neurons with vehicle, $0.5 \mu \mathrm{M}, 1 \mu \mathrm{M}$, or $10 \mu \mathrm{M}$ for $24 \mathrm{~h}$; then $40 \mu \mathrm{M}$ glutamate was added to the neurons for $24 \mathrm{~h}$. Our results showed that pretreatment with TP reduced glutamate induced neurotoxicity at a dose dependent manner as tested by MTT assay (Figure 1(b)). However, TP alone had no influence of the cell viability in the neurons (Figure 1(b)). We used $10 \mu \mathrm{M}$ $\mathrm{TP}$ in the following experiments since it had the maximal neuroprotection.

To confirm the neuroprotective effect of TP against glutamate excitotoxicity, we used TUNEL assay to measure the apoptosis. Our results showed that $40 \mu \mathrm{M}$ glutamate significantly increased the number of apoptotic cells in the primary culture cortical neurons, while pretreatment of the neurons with TP significantly reduced the cell death induced by glutamate (Figure 2 ). The above results demonstrated that $\mathrm{TP}$ protected against glutamate induced neurotoxicity in the primary culture cortical neurons.
3.2. TP Inhibited Glutamate Induced Oxidative Stress in the Neurons. We next explored the mechanism by which TP protected against glutamate excitotoxicity. We measured ROS release since it contributes to glutamate induced neuronal death. Our results confirmed that glutamate caused the production of ROS in the primary cultured cortical neurons; we further showed the pretreatment of TP inhibited the production of ROS caused by glutamate (Figure 3(a)). Moreover, we found that TP rescued the loss of SOD activity induced by glutamate in the primary cultured cortical neurons (Figure 3(b)). Thus our data demonstrated the glutamate induced oxidative stress in the neurons was rescued by TP.

3.3. Bcl-2 Mediated the Neuroprotection of Glutamate in the Neurons. The involvement of antiapoptotic protein Bcl-2 in the neuroprotection of TP against glutamate excitotoxicity in the primary cultured cortical neurons was further investigated. The cultured cortical neurons were treated with vehicle or $40 \mu \mathrm{M}$ glutamate. As shown in Figures $4(\mathrm{a})$ and $4(\mathrm{~b})$, the content of Bcl-2 in the neurons decreased significantly after treatment of glutamate compared to the control group tested by Western blot. Furthermore, pretreatment with TP rescued the glutamate induced decrease in the content of $\mathrm{Bcl}-2$ in the cultured neurons. To find out whether $\mathrm{Bcl}-2$ mediated the neuroprotection of TP in the neurons, we used siBcl-2. As shown in Figure 4(c), TP attenuated glutamate induced neurotoxicity when the neurons were treated with SiScr. In contrast, the neuroprotective effect of TP against glutamate excitotoxicity was almost completely blocked when 


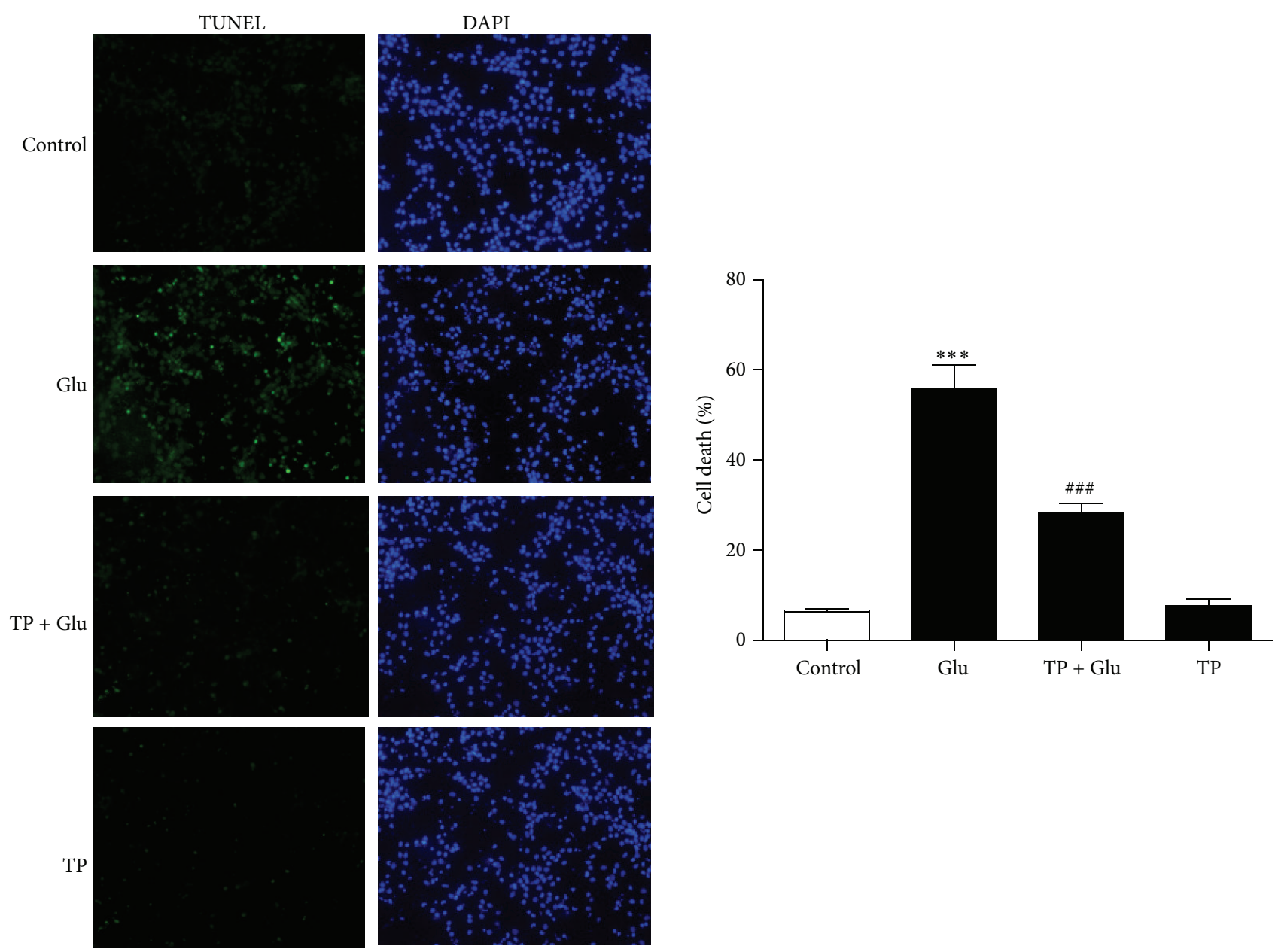

FIGURE 2: TP attenuated glutamate induced cell death in the neurons. Photomicrographs and histograms and show that TP inhibited glutamate induced apoptosis as assessed by TUNEL assay and there is no marked influence of TP alone on the number of died cells in the neurons. At least three independent experiments were performed. Data shown represent one experiment. $n=3 /$ group. One-way analysis of variance (ANOVA) followed by Tukey post hoc multiple comparisons tests, $F_{(3,8)}=58.52 .{ }^{* * *}$ compared to the control group. ${ }^{\# \#}$ compared to the Glu group.

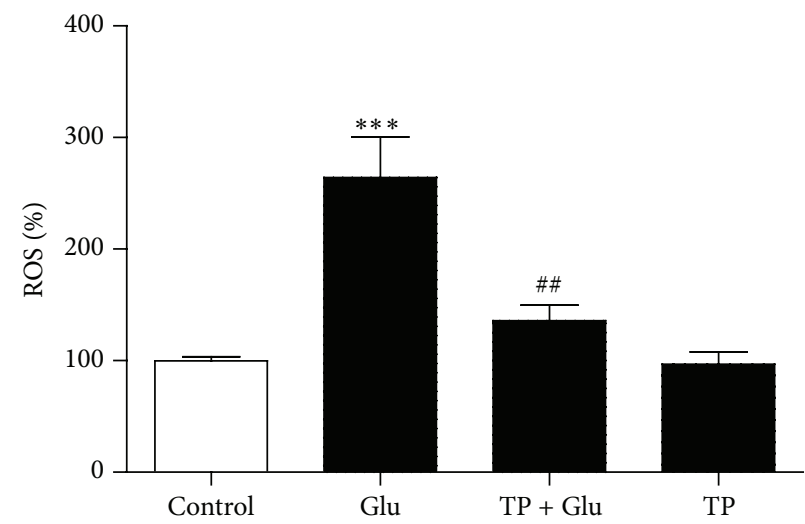

(a)

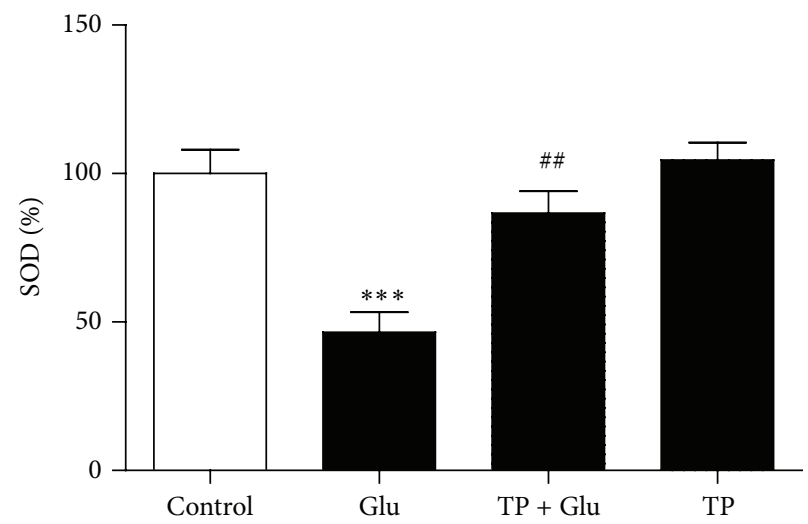

(b)

FIGURE 3: TP reduced oxidative stress induced by glutamate in the neurons. (a) Bar graphs show that glutamate induced ROS release in the primary cultured cortical neurons was prevented by incubation of TP. (b) Bar graphs show that the SOD activity in the neurons was downregulated by the glutamate, while TP treatment rescued the SOD activity. Two independent experiments were performed. Data shown represent one experiment. $n=5 /$ group. One-way analysis of variance (ANOVA) followed by Tukey post hoc multiple comparisons tests, $F_{(3,16)}$ $=14.97$ for $(\mathrm{a}) ; F_{(3,16)}=13.92$ for (b). ${ }^{* * *}$ compared to the control group and ${ }^{\# \#}$ compared to the Glu group. 


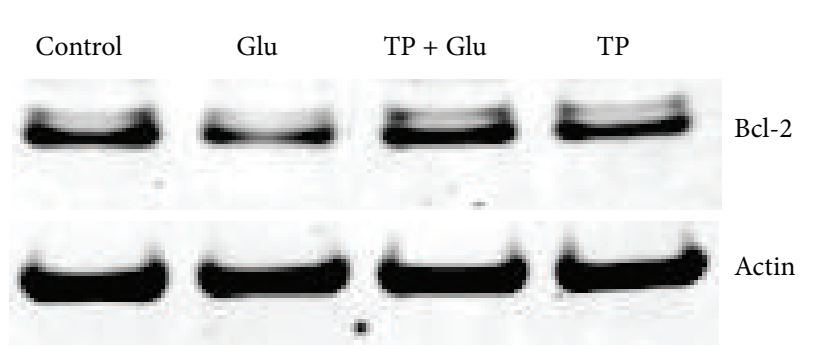

(a)

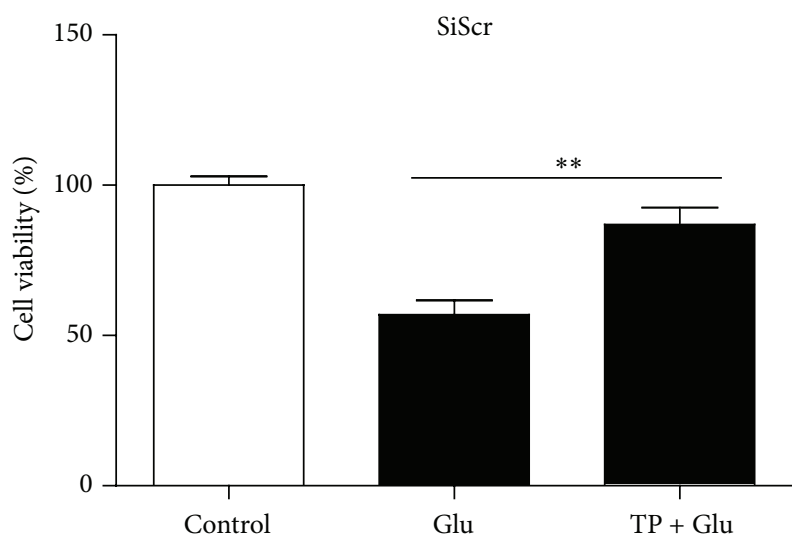

(c)

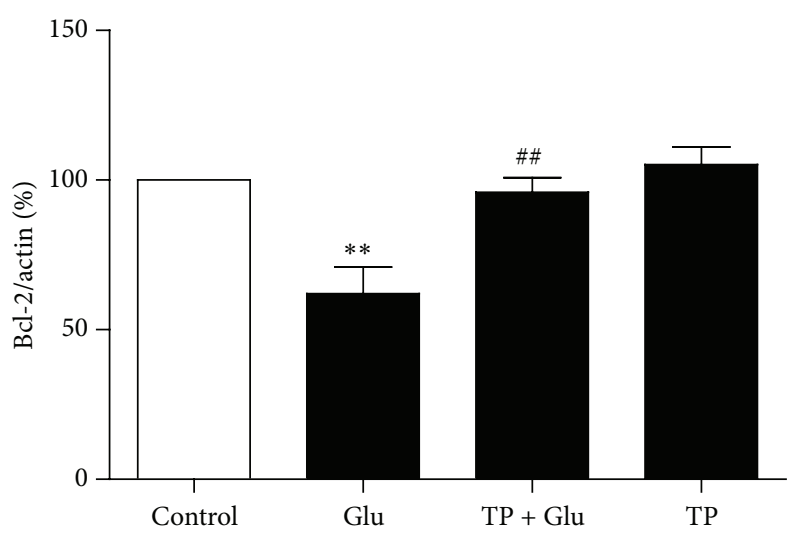

(b)

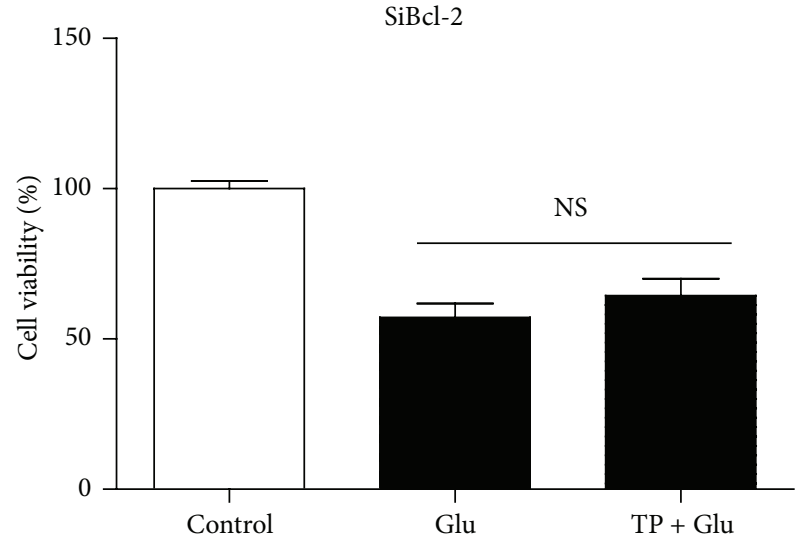

(d)

FIGURE 4: Involvement of Bcl-2 in the neuroprotection of TP. (a) Representative Western blot analysis of Bcl-2 protein in primary cultured cortical neurons after various treatments. Actin served as internal control. (b) Summary of the optical density of Bcl-2 normalized to actin. Results show that glutamate induced decrease of Bcl-2 was rescued by treatment of TP in the neurons. At least three independent experiments were performed. Data were from all the experiments combined. $n=4$ /group. One-way analysis of variance (ANOVA) followed by Tukey post hoc multiple comparisons tests, $F_{(3,12)}=11.18$. ${ }^{* *}$ compared to the control group and ${ }^{\# \#}$ compared to the Glu group. ((c), (d)) Bar graphs show that TP inhibited glutamate induced neurotoxicity in SiScr treated cortical neurons but not in SiBcl-2 treated neurons as tested by MTT assay. Two independent experiments were performed. Data shown represent one experiment. $n=5$ /group. One-way analysis of variance (ANOVA) followed by Tukey post hoc multiple comparisons tests, $F_{(2,12)}=23.19$ for $(\mathrm{c}) ; F_{(2,12)}=26.15$ for $(\mathrm{d})$.

the neurons were treated with SiBcl-2 (Figure 4(d)). Thus our data indicated that the neuroprotection of TP against glutamate induced neurotoxicity was mediated by $\mathrm{Bcl}-2$.

\subsection{Effects of TP on the Glutamate Induced Dysregulation of} Bax and Caspase-3. We also tested the proapoptotic proteins Bax and caspase-3 after various treatments. Our results showed that glutamate increased the expression of Bax in the primary cultured cortical neurons, while pretreatment with TP inhibited the glutamate induced upregulation of Bax in the neurons (Figures 5(a) and 5(b)). Moreover, we found that the glutamate induced activation of caspase- 3 was inhibited by treatment of TP in the neurons (Figures 5(c) and 5(d)). The above results suggest the involvements of Bax and caspase-3 in the neuroprotection of TP against glutamate neurotoxicity in the primary cultured cortical neurons.

\section{Discussion}

Neurodegenerative diseases are a wide class of hereditary and sporadic conditions characterized by progressive nervous system dysfunction. These disorders include Alzheimer's disease (AD), Parkinson's disease (PD), Huntington's disease (HD), and amyotrophic lateral sclerosis (ALS) which are caused by a combination of genetic and environmental factors. $\mathrm{AD}$ alone affects approximately 4.5 million Americans, projected to increase to 11 and to 16 million by 2050 [16]. With extensive research and clinical trials globally, neurodegenerative diseases remain without curative treatments, and the available therapies provide only symptom improvements. In $\mathrm{AD}$, various neurotrophic factors such as brainderived neurotrophic factor (BDNF), fibroblast growth factor 2 (FGF2), neurotrophic factor- $\alpha 1$ (NF- $\alpha 1$ ), and activitydependent neuroprotective protein (ADNP) have been studied as potential therapeutic targets for treating the malicious 


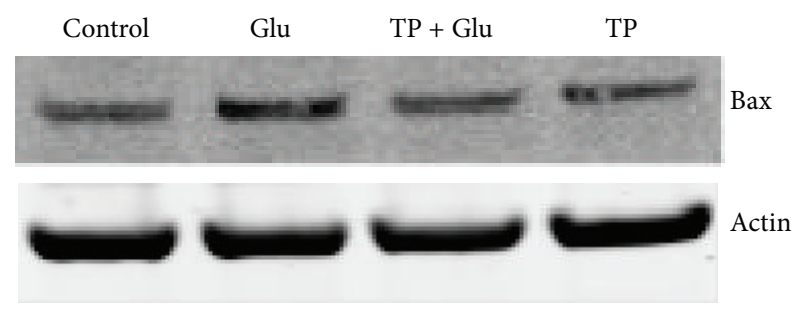

(a)

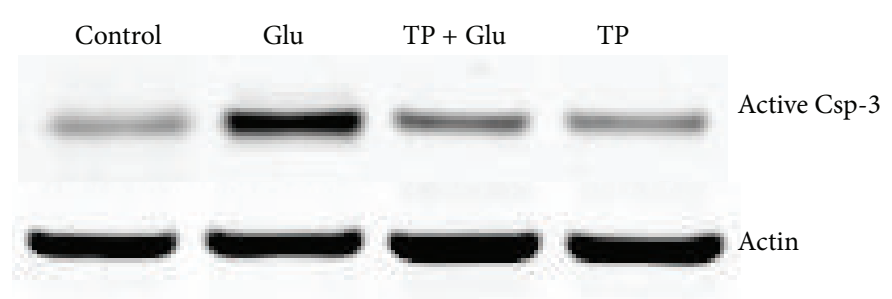

(c)

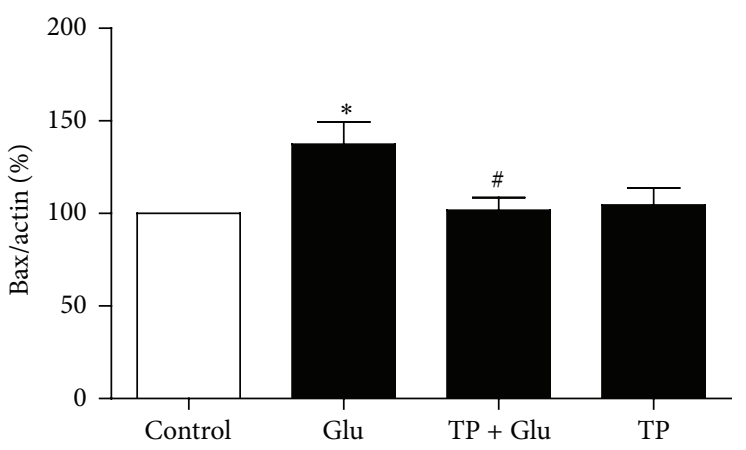

(b)

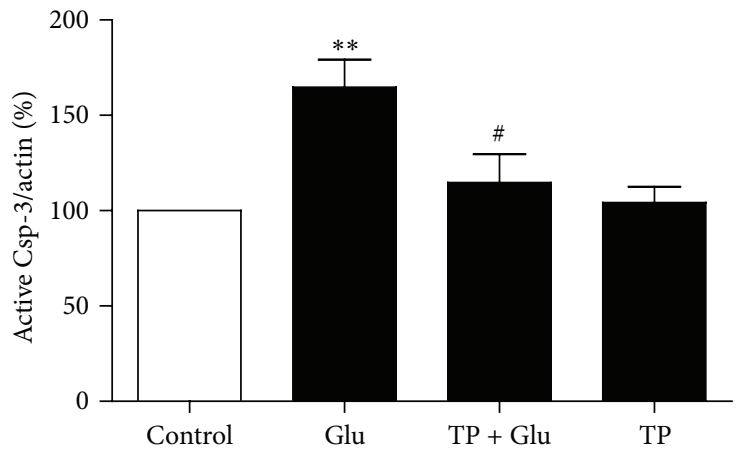

(d)

FIGURE 5: Influence of TP on glutamate induced dysregulation of Bax and caspase-3 (Csp-3). (a) Representative Western blot analysis of Bax expression in primary cultured cortical neurons after various treatments. Actin served as internal control. (b) Summary of the optical density of Bax normalized to actin. Results show that TP inhibited glutamate induced upregulation of Bax in the neurons. (c) Representative Western blot analysis of active casapse-3 in primary cultured cortical neurons after various treatments. Actin served as internal control. (d) Summary of the optical density of active caspase- 3 normalized to actin. Results show that TP inhibited glutamate induced activation of caspase- 3 in the neurons. At least three independent experiments were performed. Data were from all the experiments combined. $n=4 /$ group. Oneway analysis of variance (ANOVA) followed by Tukey post hoc multiple comparisons tests, $F_{(3,12)}=4.533$ for $(\mathrm{b}) ; F_{(3,12)}=7.162$ for $(\mathrm{d}) .{ }^{*}$ or ** compared to the control group and ${ }^{*}$ compared to the Glu group.

disease [17-20], yet there are no successful drugs developed based on these trophic factors. Thus looking for new neuroprotectants is needed for control of neurodegenerative diseases. The most suitable approaches to dissect the factors and fully study the cellular and molecular mechanisms of these diseases are global "omics" approaches. In fact, a lot of information has been gained from genomic profiling of cortical neurons following exposure to beta-amyloid [21]. Furthermore, proteomics approaches are the most innovative tools for monitoring at the proteome level the extent of protein oxidative insult and related modifications with the identification of targeted proteins [22]. Natural products such as polyphenols have now been emerging as new therapeutic targets for treating neurodegenerative diseases. In our study, we demonstrated that glutamate induced neurotoxicity was attenuated by green tea polyphenols in the primary cultured cortical neurons; our data are consistent with the previous findings of the antioxidant effect of green tea polyphenols.

Glutamate induced neuronal death is associated with the production of ROS in the neurons [23]. In this study we showed that glutamate induced ROS release in the primary cultured cortical neurons which is consistent with previous literature. Pretreatment with green tea polyphenols prevented the excessive release of ROS caused by glutamate in the neurons, confirming the neuroprotection of green tea polyphenols. We further demonstrated that the activity of the antioxidant enzyme SOD was downregulated by glutamate excitotoxicity and green tea polyphenols rescued the activity of SOD. These results suggest that the antioxidant role of green tea polyphenols might play a critical role against glutamate induced neurotoxicity.

The mitochondria have been identified as a major source of ROS, and their dysfunction with time appears to contribute to neural decay and aging [24]. Mitochondria play a key role in the regulation of apoptotic cell death and the ratio of proapoptotic to antiapoptotic Bcl-2 family members is a major checkpoint in the regulation of apoptosis [25]. The conformation of Bax is changed upon apoptosis signal; it is then translocated into the mitochondrial membranes from cytosol to form pores, which leads to the release of cytochrome $\mathrm{c}$ and subsequently activation of caspase to initiate cell death; however, the antiapoptotic protein Bcl-2 inhibits the mitochondria pore formation to prevent cell death [26]. Our data showed that glutamate downregulated the expression of Bcl2 and green tea polyphenols rescued the expression Bcl-2. Furthermore, knockdown $\mathrm{Bcl}-2$ by siRNA in the neurons 
blocked the neuroprotective property of green polyphenols against glutamate toxicity. Interestingly, it has been reported that cellular ROS levels can be decreased by the antiapoptotic protein $\mathrm{Bcl}-2$, while lower ROS levels are observed in Bcl2-expressing cells [27]. These results support the neuroprotective effect of green tea polyphenols against excitotoxicity and suggest that the recovery of mitochondria energetics may meditate the neuroprotection. We also showed that green tea polyphenols prevented the activation of caspase- 3 induced by glutamate in the neurons. This observation is consistent with caspase being downstream target of mitochondrial apoptosis.

In conclusion, we have demonstrated that green tea polyphenols protected against glutamate induced excitotoxicity in the primary cultured cortical neurons. Furthermore, we showed that the protective effect of green tea polyphenols was mediated by attenuation of oxidative stress and antimitochondrial apoptotic pathway. Thus our study supports the hypothesis that polyphenols are potential therapeutic targets for treating neurodegenerative disease; further investigations into the function of polyphenols are justified.

\section{Conflict of Interests}

The authors declare that there is no conflict of interests regarding the publication of this paper.

\section{Acknowledgments}

This work was supported by the Minzu University 985 Academic Team-Building Fund (YLDX01013, 2015MDTD13C, and 25C) and the 111 Project of China (B08044). The authors also thank Dr. Bao-Lu for his kind gift of green tea polyphenols.

\section{References}

[1] B. S. Meldrum, "Glutamate as a neurotransmitter in the brain: review of physiology and pathology," Journal of Nutrition, vol. 130, no. 4, pp. 1007S-1015S, 2000.

[2] Y. Zhou and N. C. Danbolt, "Glutamate as a neurotransmitter in the healthy brain," Journal of Neural Transmission, vol. 121, no. 8, pp. 799-817, 2014.

[3] X.-X. Dong, Y. Wang, and Z.-H. Qin, "Molecular mechanisms of excitotoxicity and their relevance to pathogenesis of neurodegenerative diseases," Acta Pharmacologica Sinica, vol. 30, no. 4, pp. 379-387, 2009.

[4] D. Nguyen, M. V. Alavi, K.-Y. Kim et al., "A new vicious cycle involving glutamate excitotoxicity, oxidative stress and mitochondrial dynamics," Cell Death \& Disease, vol. 2, no. 12, article e240, 2011.

[5] G. Olmos and J. Lladó, "Tumor necrosis factor alpha: a link between neuroinflammation and excitotoxicity," Mediators of Inflammation, vol. 2014, Article ID 861231, 12 pages, 2014.

[6] C. Parenti, A. Marrazzo, G. Aricò et al., "The antagonistic effect of the sigma 1 receptor ligand (+)-MR200 on persistent pain induced by inflammation," Inflammation Research, vol. 63, no. 3, pp. 231-237, 2014.

[7] A. Ebrahimi and H. Schluesener, "Natural polyphenols against neurodegenerative disorders: potentials and pitfalls," Ageing Research Reviews, vol. 11, no. 2, pp. 329-345, 2012.
[8] K. S. Bhullar and H. P. V. Rupasinghe, "Polyphenols: multipotent therapeutic agents in neurodegenerative diseases," Oxidative Medicine and Cellular Longevity, vol. 2013, Article ID 891748, 18 pages, 2013.

[9] F. Murray, 100 Super Supplements for a Longer Life, McGrawHill, Los Angeles, Calif, USA, 2000.

[10] X.-Y. Qin, Y. Cheng, and L.-C. Yu, "Potential protection of green tea polyphenols against intracellular amyloid beta-induced toxicity on primary cultured prefrontal cortical neurons of rats," Neuroscience Letters, vol. 513, no. 2, pp. 170-173, 2012.

[11] E. J. Okello, R. Leylabi, and G. J. McDougall, "Inhibition of acetylcholinesterase by green and white tea and their simulated intestinal metabolites," Food and Function, vol. 3, no. 6, pp. 651661, 2012.

[12] X.-Y. Qin, Y. Cheng, J. Cui, Y. Zhang, and L.-C. Yu, "Potential protection of curcumin against amyloid $\beta$-induced toxicity on cultured rat prefrontal cortical neurons," Neuroscience Letters, vol. 463, no. 2, pp. 158-161, 2009.

[13] X.-Y. Qin, Y. Cheng, and L.-C. Yu, "Potential protection of curcumin against intracellular amyloid $\beta$-induced toxicity in cultured rat prefrontal cortical neurons," Neuroscience Letters, vol. 480, no. 1, pp. 21-24, 2010.

[14] Y. Cheng and L.-C. Yu, "Galanin protects amyloid-beta-induced neurotoxicity on primary cultured hippocampal neurons of rats," Journal of Alzheimer's Disease, vol. 20, no. 4, pp. 1143-1157, 2010.

[15] Y. Cheng and L.-C. Yu, "Galanin up-regulates the expression of M1 muscarinic acetylcholine receptor via the ERK signaling pathway in primary cultured prefrontal cortical neurons," Neuroscience Letters, vol. 590, pp. 161-165, 2015.

[16] A. Melo, L. Monteiro, R. M. F. Lima, D. M. de Oliveira, M. D. de Cerqueira, and R. S. El-Bachá, "Oxidative stress in neurodegenerative diseases: mechanisms and therapeutic perspectives," Oxidative Medicine and Cellular Longevity, vol. 2011, Article ID 467180, 14 pages, 2011.

[17] Y. Cheng, N. X. Cawley, and Y. P. Loh, "Potential protection of curcumin against amyloid $\beta$-induced toxicity on cultured rat prefrontal cortical neurons," Neuroscience Bulletin, vol. 30, no. 4, pp. 692-696, 2014.

[18] T. Kiyota, K. L. Ingraham, M. T. Jacobsen, H. Xiong, and T. Ikezu, "FGF2 gene transfer restores hippocampal functions in mouse models of Alzheimer's disease and has therapeutic implications for neurocognitive disorders," Proceedings of the National Academy of Sciences of the United States of America, vol. 108, no. 49, pp. E1339-E1348, 2011.

[19] H. Koshimizu, V. Senatorov, Y. P. Loh, and I. Gozes, "Neuroprotective protein and carboxypeptidase E," Journal of Molecular Neuroscience, vol. 39, no. 1, pp. 1-8, 2009.

[20] Y. Y. Lim, V. L. Villemagne, S. M. Laws et al., "APOE and BDNF polymorphisms moderate amyloid $\beta$-related cognitive decline in preclinical Alzheimer's disease," Molecular Psychiatry, 2014.

[21] S. Paratore, R. Parenti, A. Torrisi, A. Copani, F. Cicirata, and S. Cavallaro, "Genomic profiling of cortical neurons following exposure to beta-amyloid," Genomics, vol. 88, no. 4, pp. 468479, 2006.

[22] V. Calabrese, S. Dattilo, A. Petralia et al., "Analytical approaches to the diagnosis and treatment of aging and aging-related disease: redox status and proteomics," Free Radical Research, vol. 49, no. 5, pp. 511-524, 2015. 
[23] I. J. Reynolds and T. G. Hastings, "Glutamate induces the production of reactive oxygen species in cultured forebrain neurons following NMDA receptor activation," Journal of Neuroscience, vol. 15, no. 5 I, pp. 3318-3327, 1995.

[24] S. Gandhi and A. Y. Abramov, "Mechanism of oxidative stress in neurodegeneration," Oxidative Medicine and Cellular Longevity, vol. 2012, Article ID 428010, 11 pages, 2012.

[25] E. Radi, P. Formichi, C. Battisti, and A. Federico, "Apoptosis and oxidative stress in neurodegenerative diseases," Journal of Alzheimer's Disease, vol. 42, supplement 3, pp. S125-S152, 2014.

[26] Y. Cheng, N. X. Cawley, and Y. P. Loh, "Carboxypeptidase $\mathrm{E} / \mathrm{NF} \alpha 1$ : a new neurotrophic factor against oxidative stressinduced apoptotic cell death mediated by ERK and PI3-K/AKT pathways," PLoS ONE, vol. 8, no. 8, Article ID e71578, 2013.

[27] N. Susnow, L. Zeng, D. Margineantu, and D. M. Hockenbery, "Bcl-2 family proteins as regulators of oxidative stress," Seminars in Cancer Biology, vol. 19, no. 1, pp. 42-49, 2009. 


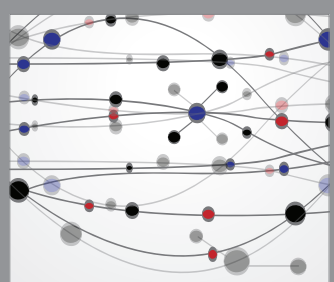

The Scientific World Journal
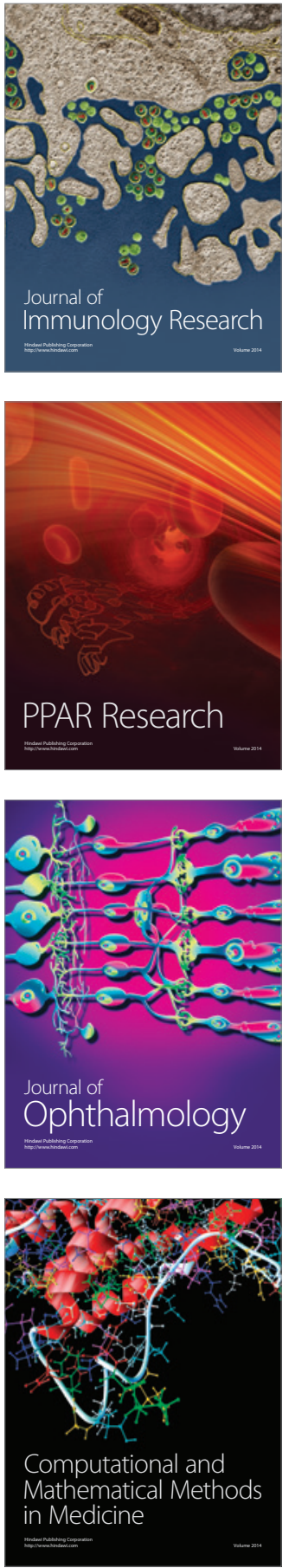

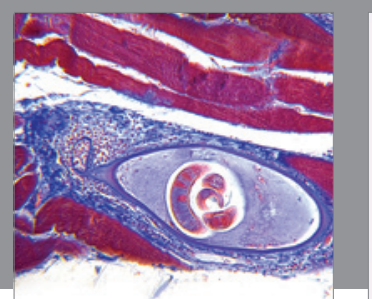

Gastroenterology Research and Practice

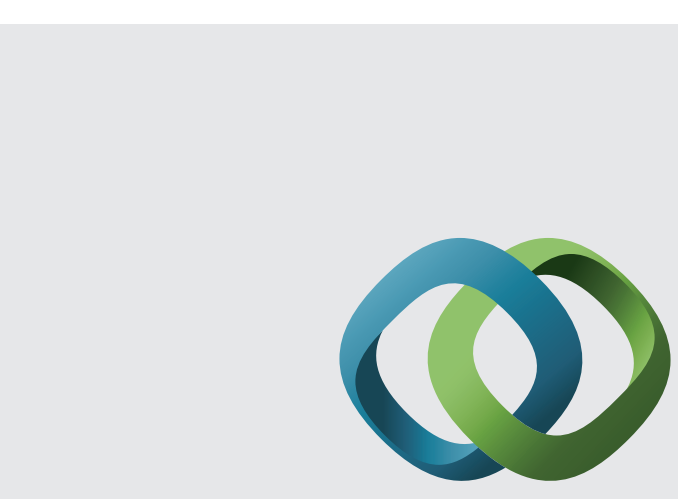

\section{Hindawi}

Submit your manuscripts at

http://www.hindawi.com
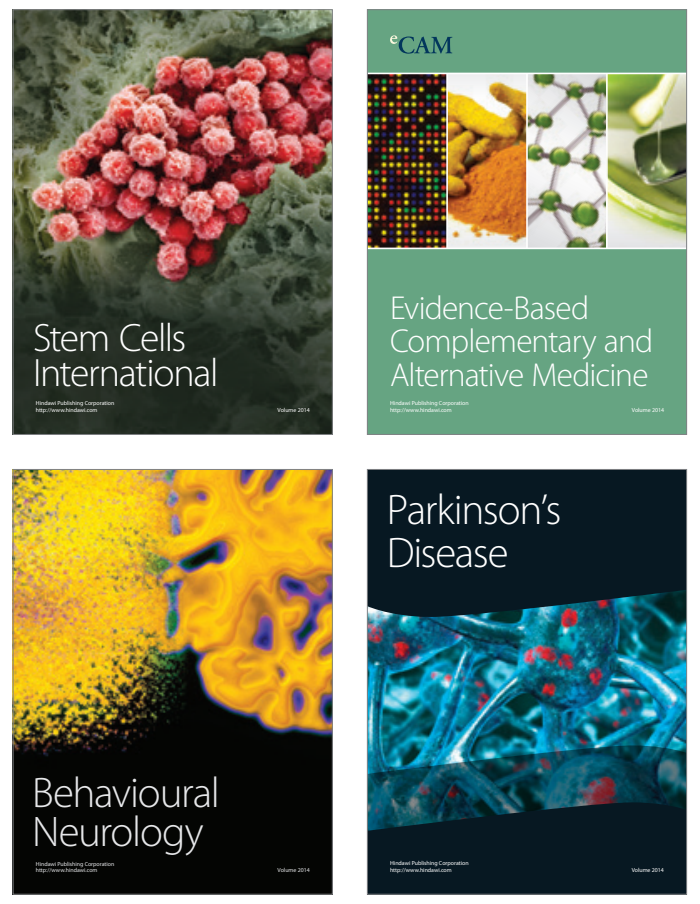
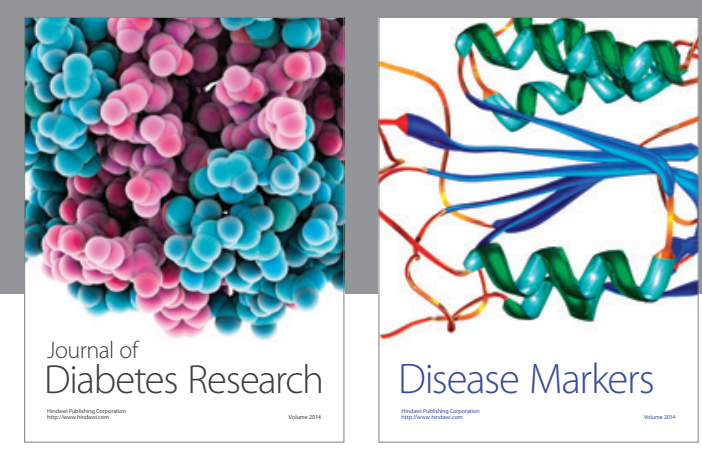

Disease Markers
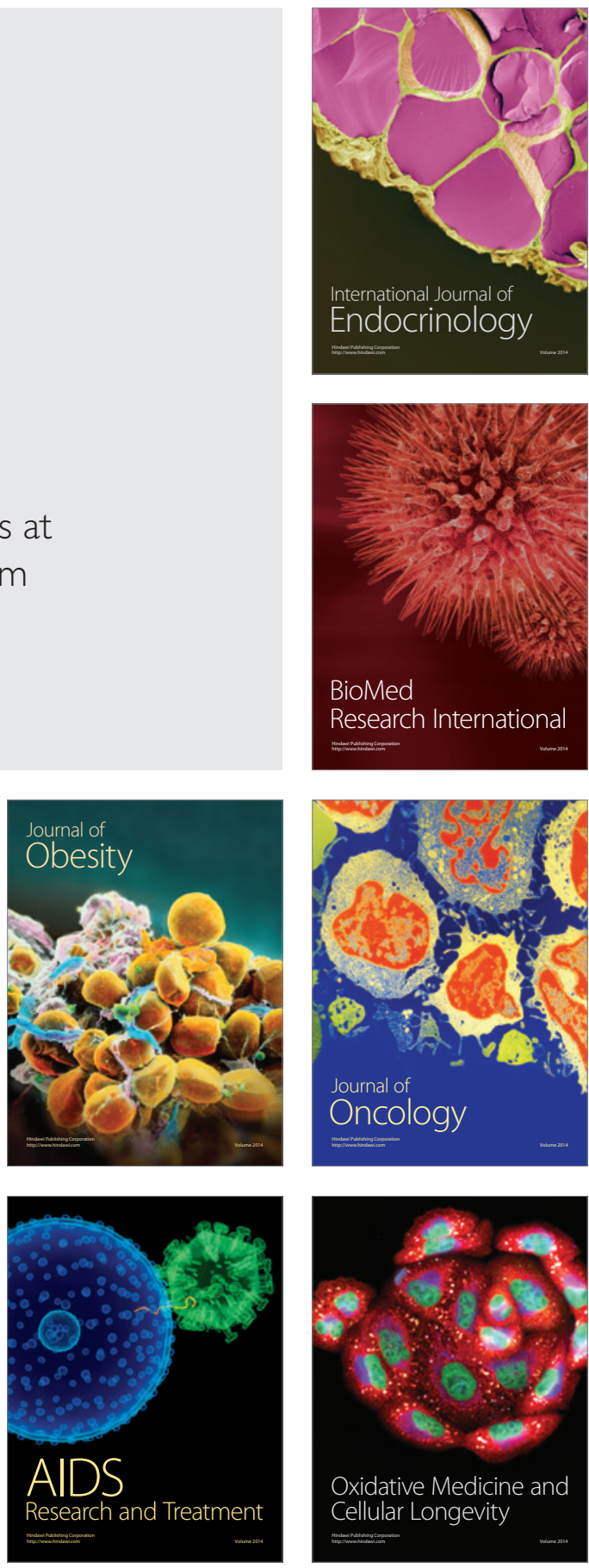